\title{
Distribution system reliability evaluation using credibility theory
}

\author{
Xufeng Xu, Joydeep Mitra* \\ Department of Electrical \& Computer Engineering, Michigan State University, USA, MI 48824 \\ *Corresponding Author: e-mail: mitra@ieee.org
}

\begin{abstract}
This paper describes a new method of using credibility theory to evaluate distribution system reliability. The advantage of this method lies in its ability to account for both objective and subjective uncertainty by integrating stochastic and fuzzy approaches. Equipment failures are modeled as random events, while the uncertainty in failure indices is modeled using credibility theory. In this manner, distribution system reliability is evaluated by simultaneously considering objective and subjective uncertainty. In this paper, a hybrid algorithm based on fuzzy simulation and Failure Mode and Effect Analysis (FMEA) is applied to determine fuzzy reliability indices of distribution system. This approach can obtain fuzzy expected values and their variances of reliability indices, and the credibilities of reliability indices meeting specified targets. The method is demonstrated using an IEEE test system comprising sixty seven nodes and thirty eight load points.
\end{abstract}

Keywords: Distribution system, reliability evaluation, credibility theory, fuzzy reliability indices, fuzzy simulation

\section{Introduction}

Distribution system reliability indices quantify, in various ways, the ability of a distribution system to meet customer demand at all its load points. Due to the larger impact of failures in the bulk power system, the development of predictive reliability approaches has received more attention from the research community. However, distribution system components are relatively more numerous and they are exposed to a more complex set of uncertain events. As distribution systems grow in complexity, with the deployment of distributed generation and storage, and of distribution automation equipment, it is important to develop more complex methods for predictive reliability analysis of distribution systems so as to suitably address these uncertainties.

Recent research efforts in distribution system reliability evaluation have focused on the following: (1) consideration of the impact of distributed generation (Atwa and Saadany, 2009; Bae and Kim, 2007) and microgrids (Bae and Kim, 2008; Kennedy, 2009); (2) incorporating weather effects (Billinton and Acharya, 2005; Billinton and Acharya, 2006); (3) time-varying failure rate (Retterath et al, 2004; Moon et al, 2004). Since all of these factors are indeterminate, some research has considered them collectively (Dong et al, 2009; Wang et al, 2002).

Uncertainty factors can be classified into two categories: stochastic factors and fuzzy factors. Usually, randomness is related to objective uncertainty factors that have statistical characteristic, while fuzziness is commonly used to define factors that have subjective uncertainty (Wang et al, 2007). Reliability evaluation methods in which only randomness is taken into account often fails to include the effect of fuzzy factors which can be significant. Models with both stochastic and fuzzy factors are often more appropriate; however, modeling this dual uncertainty can be challenging. Some recent efforts have modeled these uncertainties: trapezoidal fuzzy numbers have been used to express uncertainties in Lei et al, 2005; Yuan et al, 2007 have used interval algorithm to deal with the uncertainty of component data to calculate the interval reliability indices.

Most of fuzzy methods for reliability evaluation of distribution system are based on fuzzy set theory. In recent years researchers have found that credibility theory, which broadens the scope of fuzzy set theory, is an effective tool for representing fuzzy events, and have developed a theoretical framework similar to that of probability theory (Liu, 2004). Credibility theory has been successfully applied in power system analysis fields, such as fuzzy power flow (Wang et al, 2007) and fuzzy risk assessment (Feng et al, 2008). 
This paper focuses on the reliability evaluation of distribution system under uncertain environment and proposes an approach based on credibility theory. The approach intensively analyzes the fuzzy factors in evaluation process and defines fuzzy reliability counterparts of traditional indices. Using this approach, a hybrid algorithm based on FMEA and fuzzy simulation is developed and implemented. The algorithm is demonstrated using the distribution system connected to bus 4 of the IEEE test system commonly referred to the RBTS (Allan et al, 1991). The case study includes the computation of fuzzy expected values and variances of reliability indices, as well as ranges of these indices, which could be useful in analysis and decision making processes.

\section{Credibility Theory}

Definition 1: Let $\tilde{\xi}$ be a fuzzy variable; then the possibility, the necessity, and the credibility of the fuzzy event $\boldsymbol{A}$ are expressed as follows:

$$
\begin{aligned}
& \operatorname{Pos}\{\boldsymbol{A}\}=\sup _{x \in A} \mu_{\tilde{\xi}}(x) \\
& \operatorname{Nec}\{\boldsymbol{A}\}=1-\sup _{x \in A^{c}} \mu_{\tilde{\xi}}(x) \\
& \operatorname{Cr}\{\boldsymbol{A}\}=\frac{1}{2}(\operatorname{Pos}\{\boldsymbol{A}\}+\operatorname{Nec}\{\boldsymbol{A}\})
\end{aligned}
$$

where $\mu_{\widetilde{\xi}}(x)$ is membership function of $\widetilde{\xi} ; \boldsymbol{A}^{c}$ is the complementary event of $\boldsymbol{A}$.

In traditional fuzzy set theory, possibility measure is considered to be a parallel concept of probability measure. But it does not possess self-duality: when $\operatorname{Pos}\{\boldsymbol{A}\}=1$, fuzzy event $\boldsymbol{A}$ is not always true; when $\operatorname{Nec}\{\boldsymbol{A}\}=0, \boldsymbol{A}$ may also be true. In order to solve this problem, reference (Liu and Liu, 2002) put forward the credibility measure and established credibility theory in 2004. In this theory, credibility measure which corresponds to probability measure in probability theory possesses self-duality and monotonicity - when $\operatorname{Cr}\{\boldsymbol{A}\}=1$, fuzzy event $\boldsymbol{A}$ is inevitably true, otherwise it is false.

Definition 2 (Liu and Peng, 2005): Let $\widetilde{\xi}$ be a fuzzy variable. Then the expected value of $\xi$ is defined by

$$
E[\tilde{\xi}]=\int_{0}^{+\infty} C r\{\tilde{\xi} \geq r\} d r-\int_{-\infty}^{0} C r\{\tilde{\xi}<r\} d r
$$

provided that at least one of the two integrals is finite.

Definition 3 (Liu and Peng, 2005): Let $\widetilde{\xi}$ be a fuzzy variable with finite expected value $E[\widetilde{\xi}]$. Then the variance of $\xi$ is defined as

$$
V[\tilde{\xi}]=E\left[(\tilde{\xi}-E[\tilde{\xi}])^{2}\right]
$$

\section{The Definition of Interrelated Fuzzy Indices}

Equipment failure rate and its repair time are two basic parameters used in the reliability evaluation of distribution systems. They are statistical values with a lot of subjectivity and are also used to evaluate equipment of similar types. For this reason, this approach adopts trapezoidal fuzzy number which affords comparatively broad adaptability in engineering area.

\subsection{Modeling of fuzzy factors}

1) Failure rate $\tilde{\lambda}_{i}$

The equipment failure rate is generally used to quantify the probability of failure of a specific piece of equipment. Although equipment status in the future is not predictable, the failure rate of this equipment can be obtained from tests or experience. Also, $\tilde{\lambda}_{i}$ can be modeled by trapezoidal fuzzy number $\tilde{\lambda}_{i}=\left(\lambda_{i}^{1}, \lambda_{i}^{2}, \lambda_{i}^{3}, \lambda_{i}^{4}\right)$, in which $\left[\lambda_{i}^{1}, \lambda_{i}^{4}\right]$ is the failure rate interval of specific equipment $i$ and $\left[\lambda_{i}^{2}, \lambda_{i}^{3}\right]$ is the most possible limits. The membership function of $\tilde{\lambda}_{i}$ is defined as below:

$$
\mu_{\tilde{\lambda}_{i}}(x)=\left\{\begin{array}{cl}
\frac{x-\lambda_{i}^{1}}{\lambda_{i}^{2}-\lambda_{i}^{1}}, & \lambda_{i}^{1} \leq x \leq \lambda_{i}^{2} \\
1, & \lambda_{i}^{2}<x<\lambda_{i}^{3} \\
\frac{\lambda_{i}^{4}-x}{\lambda_{i}^{4}-\lambda_{i}^{3}}, & \lambda_{i}^{3} \leq x \leq \lambda_{i}^{4} \\
0, & x<\lambda_{i}^{1} \text { or } x>\lambda_{i}^{4}
\end{array}\right.
$$




\section{2) Repair time $\widetilde{r}_{i}$}

Since the insufficiency of information will result the inaccuracies of forecasting failure time, for the equipment $i$, trapezoidal fuzzy number $\widetilde{r}_{i}=\left(r_{i}^{1}, r_{i}^{2}, r_{i}^{3}, r_{i}^{4}\right)$ is used to describe repair time because this number is more reasonable than point value, and its membership function $\mu_{\widetilde{r_{i}}}$ is shown in Figure 1.

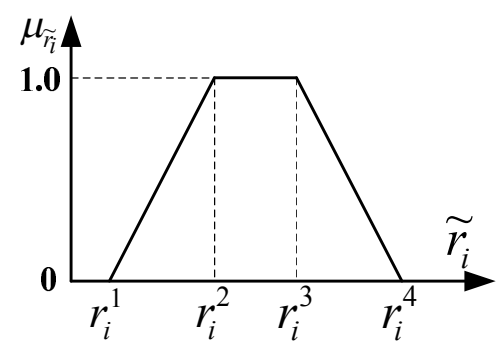

Figure 1. Membership function of repair time

\subsection{Fuzzy reliability indices}

Distribution networks are often radial and consist of series connections of lines, transformers, isolating switches, fuses, etc. Service continuity requires each equipment between power resource and load to be online. Therefore, the effect of the series system failure rate $\tilde{\lambda}_{s}$, repair time $\widetilde{r}_{s}$ and time to failure (up time) $\widetilde{T}_{s}$ are taken into account as follows:

$$
\begin{aligned}
& \tilde{\lambda}_{s}=\sum \tilde{\lambda}_{i} \\
& \tilde{r}_{s}=\frac{\sum \tilde{\lambda}_{i} \tilde{r}_{i}}{\sum \tilde{\lambda}_{i}} \\
& \tilde{T}_{s}=\frac{\tilde{\lambda}_{s}}{\tilde{\lambda}_{s}+1 / \widetilde{r}_{s}} \approx \sum \tilde{\lambda}_{i} \tilde{r}_{i}
\end{aligned}
$$

where $\tilde{\lambda}_{i}, \tilde{r}_{i}$ are failure rate and repair time of equipment $i$ respectively. Subscript $s$ expresses equivalent series network from power sending end to the receiving end.

The definition of fuzzy reliability indices of distribution system is given as follows, based on Billinton and Allan, 1996. Comparing with regular standard, those fuzzy indices indicate the impact of fuzzy parameters on the reliability indices.

1) Fuzzy Energy Not Supplied (ENS)

$$
\mathrm{ENS}=\sum d_{s} \widetilde{T}_{s}(\mathrm{MWh} / \mathrm{yr})
$$

where $d_{s}$ is the load of the equivalent series network. As $\tilde{\lambda}_{i}$ and $\tilde{r}_{i}$ are fuzzy variables, ENS is also a fuzzy index reflecting the influence of parameter ambiguity on reliability index.

2) System Average Interruption Frequency Index (SAIFI)

$$
\text { SAIFI }=\frac{\sum \tilde{\lambda}_{s} N_{s}}{\sum N_{s}} \text { (int/cus-yr) }
$$

where $N_{s}$ is the number of users on load node $s$.

3) System Average Interruption Duration Index (SAIDI)

$$
\mathrm{SAIDI}=\frac{\sum \widetilde{T}_{s} N_{s}}{\sum N_{s}}(\mathrm{hr} / \text { cus-yr })
$$

4) Customer Average Interruption Duration Index (CAIDI)

$$
\text { CAIDI }=\frac{\sum \widetilde{T}_{s} N_{s}}{\sum \widetilde{\lambda}_{s} N_{s}}(\mathrm{hr} / \mathrm{int})
$$


5) Average Service Availability Index (ASAI)

$$
\mathrm{ASAI}=\frac{\sum 8760 \times N_{s}-\sum \widetilde{T}_{s} N_{s}}{\sum 8760 \times N_{s}}
$$

6) Average Energy Not Supplied (AENS)

$$
\mathrm{AENS}=\frac{\sum \widetilde{T}_{s} d_{s}}{\sum N_{s}}(\mathrm{MWh} / \text { cus-yr })
$$

\section{Solution Procedure}

This approach adopts Failure mode and effect analysis (FMEA) (Billinton and Allan, 1996) to evaluate reliability. The entire process based on fuzzy sampling can be divided into three main parts and it is described as follows.

\subsection{Distribution system analysis}

First, the branch set connected by closed switches is defined as Sub-Power Supply System (SPSS). Based on the status of switches, the distribution system can be divided into multiple SPSS, which are connected with tie switches. For example, SPSS corresponding to feeder F1 in Figure 2 is composed of $\{1,2, \cdots, 12\}$.

Secondly, based on the definition of SPSS, the power supply area of each feeder is analyzed by depth-first search strategy. The searching process begins from power source and ends in load point or tie-line.

Finally, two matrices are defined and generated: one is network layer matrix which is used to reflect the level of branches and their relationships; and the other is loading point information matrix which contains information of each load point, such as number, active load values, number of customers, branch numbers and feeder numbers.

\subsection{Reliability evaluation process}

The procedure for evaluating fuzzy reliability indices consists of the following steps.

Step 1: Data input:

Set $v_{i}=0(i=1,2, \cdots, 6)$ and fuzzy simulation time $N$.

Step 2: Fuzzy sampling:

Randomly generating $\lambda^{k}=\left(\lambda_{1}^{\prime}, \lambda_{2}^{\prime}, \cdots \lambda_{n}^{\prime}\right)$ from $\varepsilon$ level cut sets of fuzzy vector $\tilde{\lambda}$. The membership degree of $\lambda^{k}$ can be obtained by following equation:

$$
v_{\lambda}^{k}=\mu_{\widetilde{\lambda}_{1}}\left(\lambda_{1}^{\prime}\right) \cap \mu_{\widetilde{\lambda}_{2}}\left(\lambda_{2}^{\prime}\right) \cap \cdots \mu_{\widetilde{\lambda}_{n}}\left(\lambda_{n}^{\prime}\right)
$$

where $\varepsilon$ is a positive number small enough; $n$ is equipment number; $k=1,2, \cdots, N$.

Similarly, fuzzy random value of $\tilde{\boldsymbol{r}}$ is $\boldsymbol{r}^{k}$, and its membership degree is $v_{\boldsymbol{r}}^{k}$.

Step 3: Calculate reliability indices of load point using (7), (8) and (9). Input data required are network layer matrix, load point information matrix and fuzzy sampling values.

The network reliability is assessed as well as reliability index $f_{i}^{k}\left(\lambda^{k}, \boldsymbol{r}^{k}\right)$ using (10)-(15).

Step 4: Calculate $f_{i}^{k}\left(\lambda^{k}, \boldsymbol{r}^{k}\right)$, and its membership degree $v^{k}=v_{d}^{k} \cap v_{\lambda}^{k}$.

Step 5: Repeat steps 2-4 for a total of $N$ times;

Step 6: Calculate $a_{i}$ and $b_{i}$ by the following equations, where $a_{i}$ and $b_{i}$ are both non-negative real numbers.

$$
\begin{aligned}
& a_{i}=f_{i}^{1}\left(\lambda^{1}, \boldsymbol{r}^{1}\right) \cap f_{i}^{2}\left(\lambda^{2}, \boldsymbol{r}^{2}\right) \cdots \cap f_{i}^{N}\left(\lambda^{N}, \boldsymbol{r}^{N}\right) \\
& b_{i}=f_{i}^{1}\left(\lambda^{1}, \boldsymbol{r}^{1}\right) \cup f_{i}^{2}\left(\lambda^{2}, \boldsymbol{r}^{2}\right) \cdots \cup f_{i}^{N}\left(\lambda^{N}, \boldsymbol{r}^{N}\right)
\end{aligned}
$$

Step 7: Generate $\tau_{i}$ from $\left[a_{i}, b_{i}\right]$ randomly.

Step 8: Set $v_{i} \leftarrow v_{i}+\operatorname{Cr}\left\{f_{i}(\tilde{\lambda}, \tilde{\boldsymbol{r}}) \geq \tau_{i}\right\}$.

$\operatorname{Cr}\left\{f_{i}(\tilde{\lambda}, \widetilde{\boldsymbol{r}}) \geq \tau_{i}\right\}$ are approximately calculated using (19).

$$
\operatorname{Cr}\left\{f_{i}(\tilde{\lambda}, \widetilde{\boldsymbol{r}}) \geq \tau_{i}\right\}=\frac{1}{2}\left(\max _{1 \leq k \leq N}\left\{v^{k} \mid f_{i}^{k}\left(\lambda^{k}, \boldsymbol{r}^{k}\right) \geq \tau_{i}\right\}+\min _{1 \leq k \leq N}\left\{1-v^{k} \mid f_{i}^{k}\left(\lambda^{k}, \boldsymbol{r}^{k}\right)<\tau_{i}\right\}\right)
$$

Step 9: Repeat steps 7,8 for a total of $N^{\prime}$ times; $N^{\prime}$ is a given positive integer.

Step 10: $E\left[f_{i}(\tilde{\lambda}, \tilde{\boldsymbol{r}})\right]=a_{i}+v_{i} \cdot\left(b_{i}-a_{i}\right) / N^{\prime},(i=1,2, \cdots, 6)$. 
Fuzzy reliability indices $V\left[f_{i}(\tilde{\lambda}, \tilde{\boldsymbol{r}})\right]$ can used to reflect the rate of deviation between fuzzy sampling values and fuzzy expected value, just as the definition of variance in probability theory. The deriving of variance of fuzzy variable is basically the same as the above process; the difference lies in the calculation of fuzzy sampling value of variance $g_{i}^{k}\left(\lambda^{k}, \boldsymbol{r}^{k}\right)$ which can be obtained from (20).

$$
g_{i}^{k}\left(\lambda^{k}, \boldsymbol{r}^{k}\right)=\left(f_{i}^{k}\left(\lambda^{k}, \boldsymbol{r}^{k}\right)-E\left[f_{i}(\tilde{\lambda}, \widetilde{\boldsymbol{r}})\right)^{2}\right.
$$

\subsection{Credibility calculation}

Sometimes it may be necessary, during planning or operation, to use credibility of reliability indices meeting some stipulated levels or guarantees; this credibility can be obtained by following equation:

$$
\operatorname{Cr}\left\{f_{i}(\tilde{\lambda}, \tilde{\boldsymbol{r}}) \leq F_{i}\right\}=\frac{1}{2}\left(\max _{1 \leq k \leq N}\left\{v^{k} \mid f_{i}^{k}\left(\lambda^{k}, \boldsymbol{r}^{k}\right) \leq F_{i}\right\}+\min _{1 \leq k \leq N}\left\{1-v^{k} \mid f_{i}^{k}\left(\lambda^{k}, \boldsymbol{r}^{k}\right)>F_{i}\right\}\right)
$$

where $F_{i}(i=1,2, \cdots, 6)$ is default value which is also the value expected.

\section{Simulation Results}

The IEEE-RBTS Bus 4 system (Allan et al, 1991) is used to demonstrate the feasibility of the proposed model and algorithm. A one-line diagram of the system is shown in Figure 2. This system contains 67 nodes (including 38 load nodes), 71 lines and 26 switches. The main feeders are LGJ-240, trunk lines are LGJ-120, and branch lines are LGJ-50. Parameters used in the algorithm are as follows: $N=5000 ; N^{N}=2000$. The equipment failure rate and repair time are fuzzified by using the multiplying factors $[0.80,0.95,1.05,1.2]$.

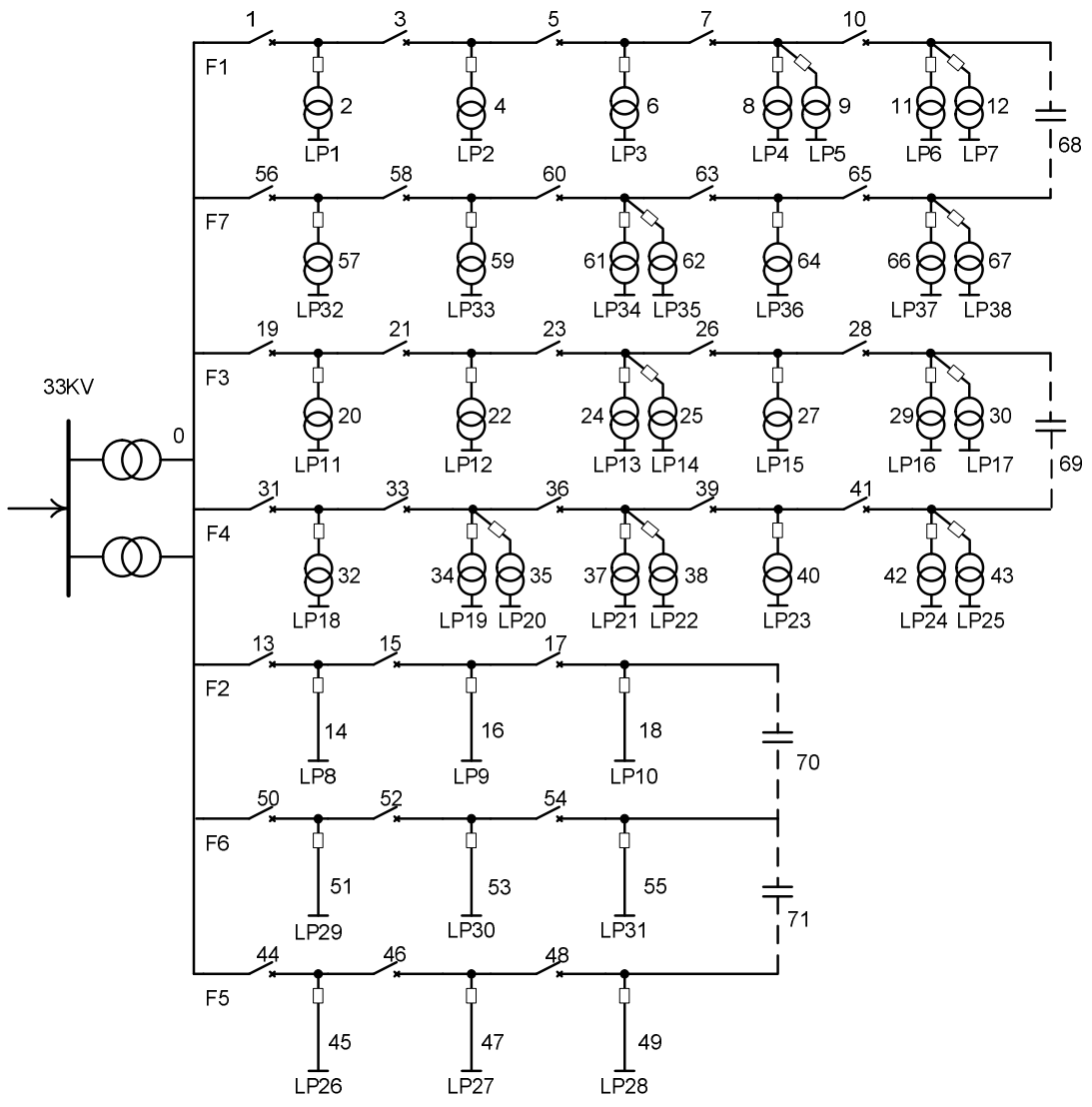

Figure 2. Distribution system for RBTS Bus 4

This work analyzes the scenario that includes disconnects, fuses and alternative supply. The fuzzy expected value of reliability indices are shown in the column three of Table 1. The result derived from the approach in this work compare well with results reported in Allan and Billinton, 1991. Fuzzy reliability indices of feeder are shown in Table 2, and the fuzzy expected values and variances of three main reliability indices of each load points are shown in Figure 3, Figure $\mathbf{4}$ and Figure 5. The stars denote the fuzzy expected values while the circles denote the traditional reliability indices. 
Table 1. Reliability indices of the system

\begin{tabular}{|c|c|c|c|}
\hline Reliability indices & $\begin{array}{c}\text { Result reported in Allan } \\
\text { and Billinton, 1991 }\end{array}$ & $\begin{array}{c}\text { Result obtained using } \\
\text { proposed method }\end{array}$ & $\begin{array}{c}\text { Deviation } \\
(\%)\end{array}$ \\
\hline SAIFI (int/cus-yr) & 0.300 & 0.2993 & 0.2331 \\
\hline SAIDI (hr/cus-yr) & 3.47 & 3.4694 & 0.0184 \\
\hline CAIDI (hr/int) & 11.56 & 11.6412 & 0.7025 \\
\hline ASAI & 0.9996 & 0.9996 & 0.0000 \\
\hline ENS (MWh/yr) & 54.293 & 54.3161 & 0.0425 \\
\hline AENS (MWh/cus-yr) & 11.36 & 11.3655 & 0.0481 \\
\hline
\end{tabular}

Table 2. Feeder fuzzy reliability indices

\begin{tabular}{|c|c|c|c|c|c|c|}
\hline Feeders & $\begin{array}{c}\text { SAIFI } \\
\text { (int./cus.yr) }\end{array}$ & $\begin{array}{c}\text { SAIDI } \\
\text { (hr/cus.yr) }\end{array}$ & $\begin{array}{c}\text { CAIDI } \\
\text { (hr/int.) }\end{array}$ & ASAI & $\begin{array}{c}\text { ENS } \\
\text { (MWh/yr) }\end{array}$ & $\begin{array}{c}\text { AENS } \\
\text { (MWh/cus.yr) }\end{array}$ \\
\hline F1 & 0.3027 & 3.4707 & 11.6110 & 0.9996 & 12.1958 & 11.0857 \\
\hline F2 & 0.1888 & 0.3805 & 2.0120 & 1.0000 & 1.3277 & 443.8710 \\
\hline F3 & 0.2933 & 3.4698 & 12.0096 & 0.9996 & 12.0032 & 11.1142 \\
\hline F4 & 0.3070 & 3.4766 & 11.4866 & 0.9996 & 13.9398 & 10.7227 \\
\hline F5 & 0.1860 & 0.3763 & 2.0332 & 1.0000 & 1.1309 & 376.6532 \\
\hline F6 & 0.1940 & 0.3657 & 1.8882 & 1.0000 & 1.2704 & 423.0530 \\
\hline F7 & 0.2950 & 3.4722 & 11.8993 & 0.9996 & 12.4705 & 9.6672 \\
\hline
\end{tabular}

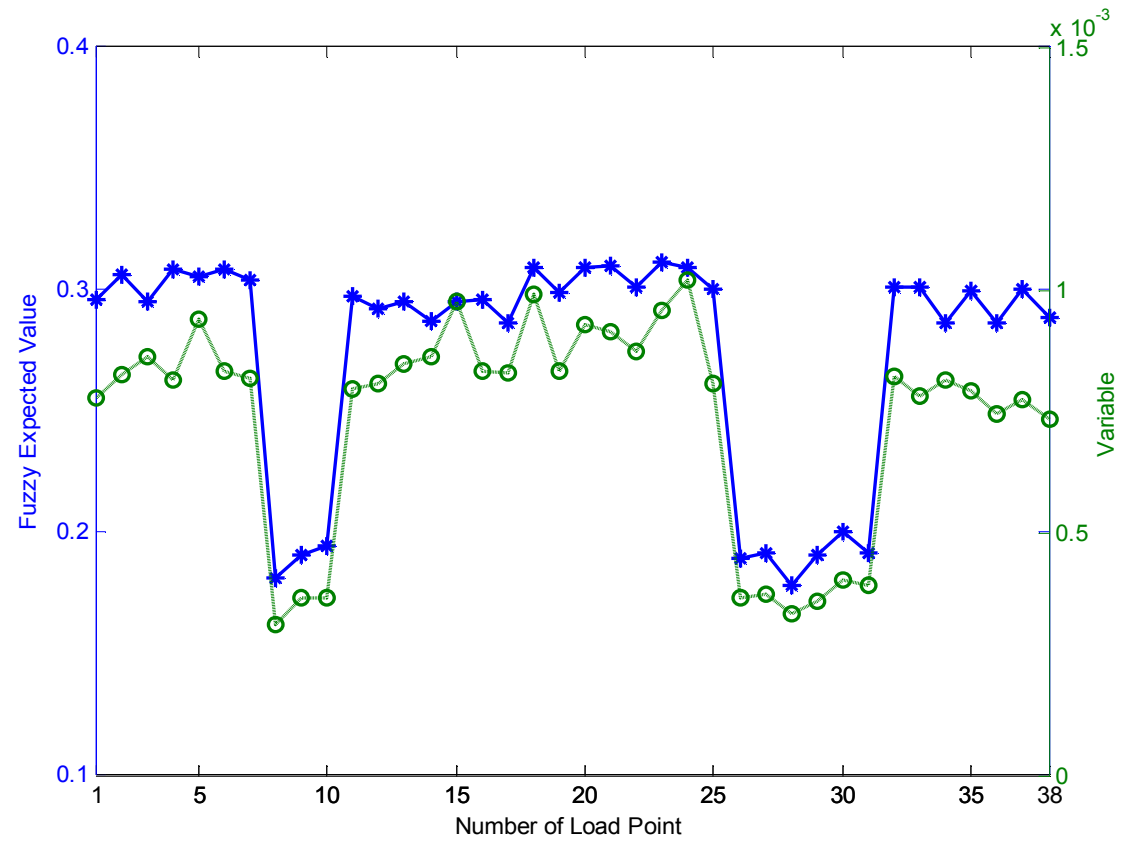

Figure 3. Failure rates of load points 


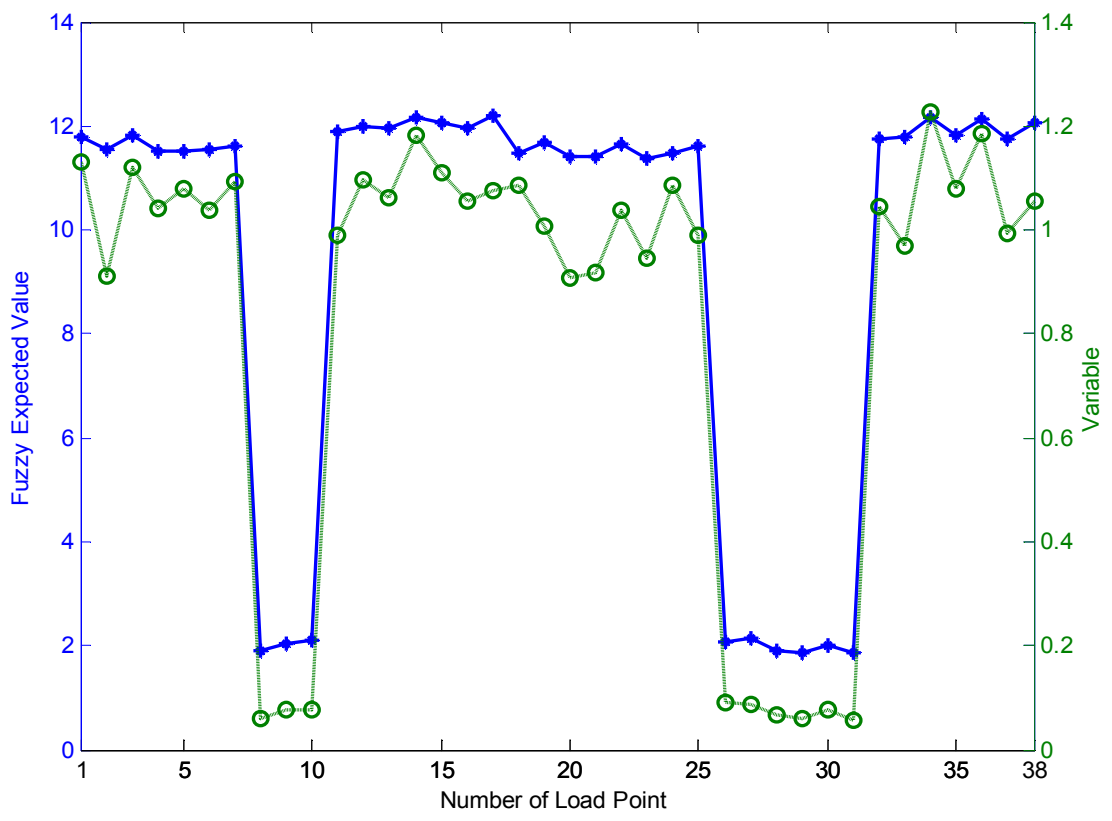

Figure 4. The average interruption durations of load points

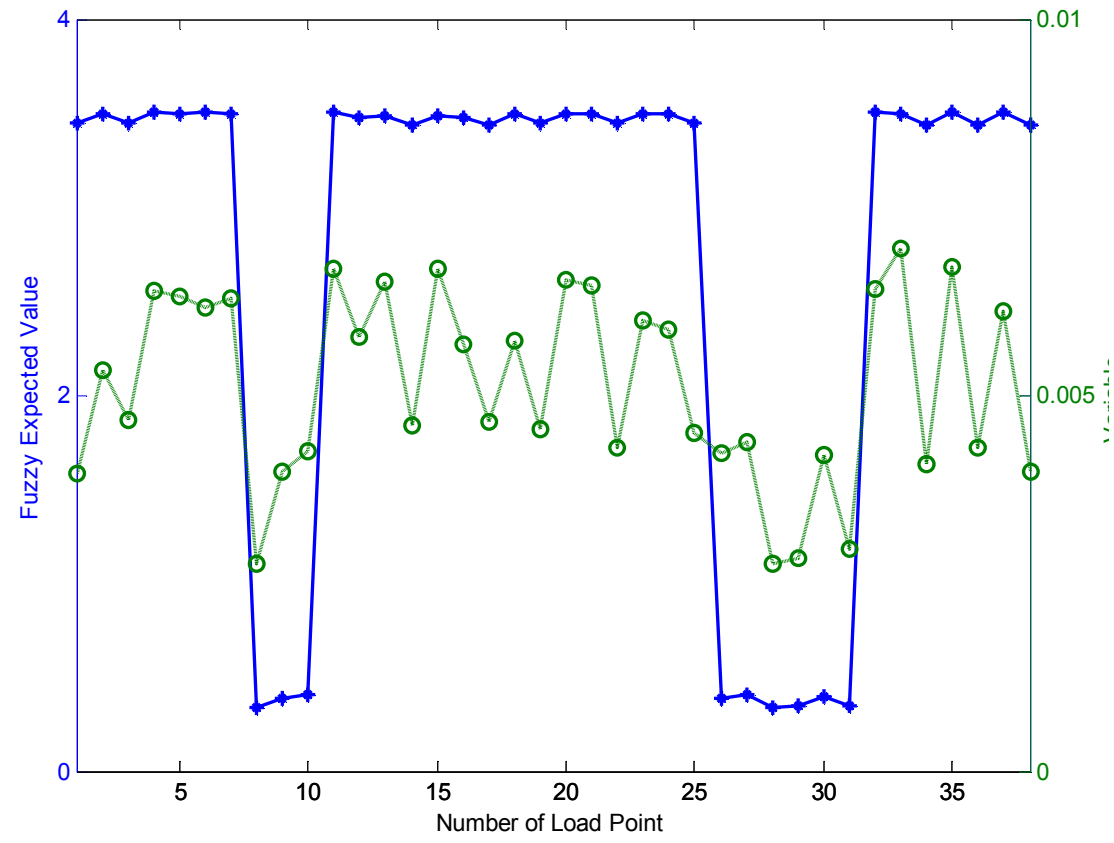

Figure 5. The failure time of load points

The fuzzy expected value $E\left[f_{E N S}(\tilde{\lambda}, \widetilde{\boldsymbol{r}})\right]$ and its variable $\left.V \mid f_{E N S}(\tilde{\lambda}, \widetilde{\boldsymbol{r}})\right]$ of ENS is $54.3161 \mathrm{MWh} / \mathrm{yr}$ and 0.2676 respectively. If we set 0.005 as unit interval, the range of fuzzy sampling value of ENS can be divided into several intervals. Defining $m_{j}$ as the frequency that sampling value will be inside band $j$ and $\frac{m_{j}}{N}$ as the subjection frequency function, the relationship between $\frac{m_{j}}{N}$ and sampling value ENS is shown in Figure 6. It can be seen that most of sampling values assemble around $E\left[f_{E N S}(\tilde{\lambda}, \tilde{\boldsymbol{r}})\right]$. In order to reflect the degree of dispersion, unbiased variance $\sigma$ is defined by square root of variance $\left.V \mid f_{E N S}(\tilde{\lambda}, \tilde{\boldsymbol{r}})\right]$. Within the interval $[53.7988,54.8334]([E-\sigma, E+\sigma])$, the total percent of frequency is 0.9936 . 


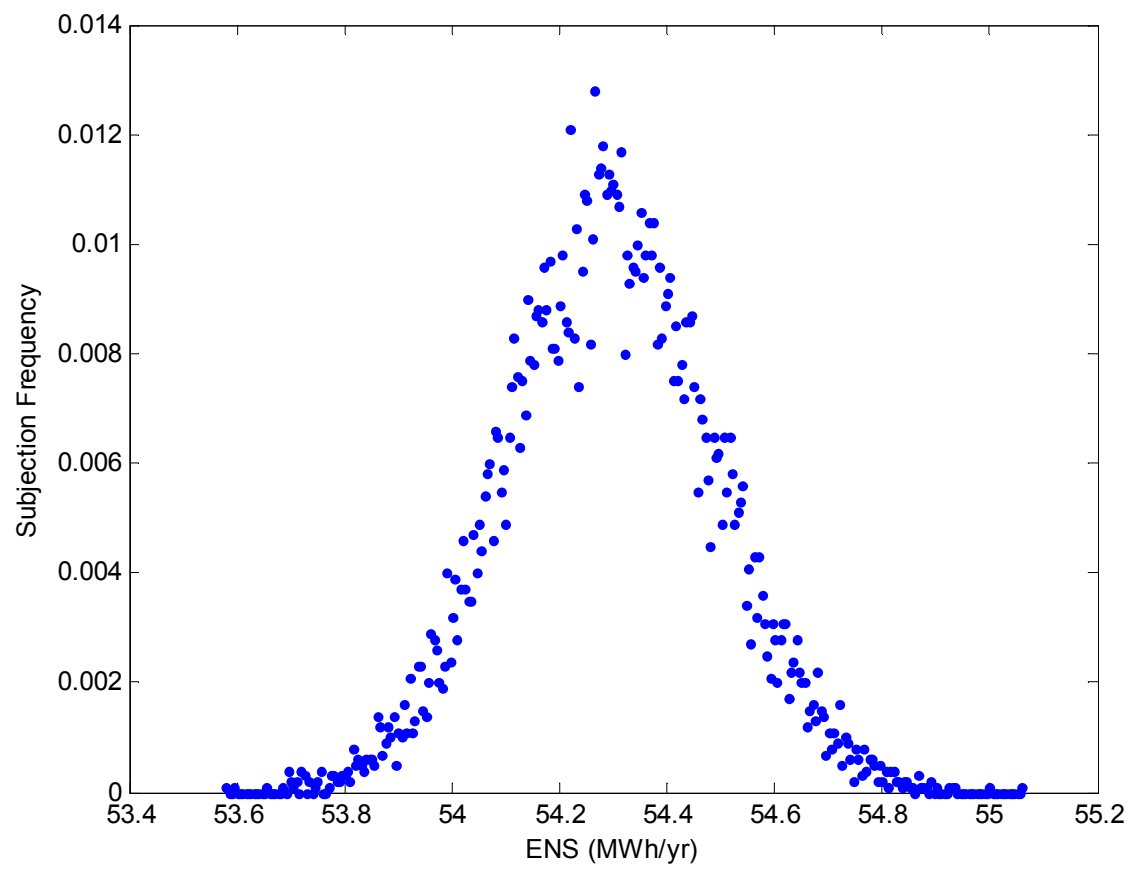

Figure 6. Frequency distribution of simulation results of ENS

In order to evaluate the influence of uncertain parameters on final results, three cases are designed for comparison: Case 1, using parameters given; Case 2, fuzzifying multiplying factor is [0.70, 0.95, 1.05, 1.3]; Case 3, uses a triangular membership function, with fuzzifying multiplying factor is $[0.8,1.0,1.2]$. The results are shown in Table 3. It can be found that the influence of uncertainty on fuzzy variance will be much more than fuzzy expected value, through comparing Case 1 and Case 2. Triangle fuzzy number which is also widely used in engineering is adopted in Case 3.

Table 3. Fuzzy expected value and variance of system reliability indices

\begin{tabular}{|c|c|c|c|c|c|c|c|}
\hline \multicolumn{2}{|c|}{ Indices } & $\begin{array}{c}\text { SAIFI } \\
\text { (int./cus.yr) }\end{array}$ & $\begin{array}{c}\text { SAIDI } \\
\text { (hr/cus.yr) }\end{array}$ & $\begin{array}{c}\text { CAIDI } \\
\text { (hr/int.) }\end{array}$ & ASAI & $\begin{array}{c}\text { ENS } \\
(\mathrm{MWh} / \mathrm{yr})\end{array}$ & $\begin{array}{c}\text { AENS } \\
(\mathrm{MWh} / \mathrm{cus} . \mathrm{yr})\end{array}$ \\
\hline \multirow{3}{*}{ Case1 } & $E\left[f_{i}\right]$ & 0.2993 & 3.4694 & 11.6412 & 0.9996 & 54.3161 & 11.3655 \\
\cline { 2 - 8 } & $V\left[f_{i}\right]$ & 0.0002 & 0.0007 & 0.3224 & 0.0000 & 0.2676 & 0.0117 \\
\hline \multirow{3}{*}{ Case2 } & $E\left[f_{i}\right]$ & 0.3008 & 3.4706 & 11.6695 & 0.9996 & 54.3579 & 11.3744 \\
\cline { 2 - 9 } & $V\left[f_{i}\right]$ & 0.0006 & 0.0018 & 0.8139 & 0.0000 & 0.6190 & 0.0271 \\
\hline \multirow{2}{*}{ Case3 } & $E\left[f_{i}\right]$ & 0.3007 & 3.4706 & 11.5725 & 0.9996 & 54.3344 & 11.3693 \\
\cline { 2 - 9 } & $V\left[f_{i}\right]$ & 0.0003 & 0.0008 & 0.3408 & 0.0000 & 0.3311 & 0.0145 \\
\hline
\end{tabular}

In our approach, equipment failure rate and repair time are uncertain, so reliability indices will also not assured. Thus, credibility is also a good option in addition to fuzzy expected value of reliability indices. We design another two cases: Case $4, F_{1}=0.32, F_{2}=3.5, F_{3}=12.3, F_{4}=0.9996, F_{5}=58, F_{6}=12.15$; Case 5, assigning the reference result in column two of Table 2 to $F_{i}(i=1,2, \cdots 6)$ respectively. The simulation results are shown in Table 4. It can be seen that the higher the demand on the reliability indices, the lower credibility will be. The relationship between $\operatorname{Cr}\left\{f_{\text {SAIFI }}<F_{\text {SAIFI }}\right\}$ and $F_{\text {SAIFI }}$ is shown in Figure 7 and the curve shape is dependent on the fuzzy expression of parameters. The relationships of another five reliability indices are shown in Figure 8, Figure 9, Figure 10, Figure 11 and Figure 12 respectively. 
Table 4. Credibility of reliability indices

\begin{tabular}{|c|l|l|}
\hline Indices & \multicolumn{1}{|c|}{ Case4 } & \multicolumn{1}{c|}{ Case5 } \\
\hline SAIFI & $\operatorname{Cr}\left\{f_{1}<0.32\right\}=0.9773$ & $\operatorname{Cr}\left\{f_{1}<0.3\right\}=0.0844$ \\
\hline SAIDI & $\operatorname{Cr}\left\{f_{2}<3.5\right\}=0.9904$ & $\operatorname{Cr}\left\{f_{2}<3.47\right\}=0.0333$ \\
\hline CAIDI & $\operatorname{Cr}\left\{f_{3}<12.3\right\}=0.9900$ & $\operatorname{Cr}\left\{f_{3}<11.56\right\}=0.9667$ \\
\hline ASAI & $\operatorname{Cr}\left\{f_{4}>0.9996\right\}=0.9990$ & $\operatorname{Cr}\left\{f_{4}>0.9996\right\}=0.9990$ \\
\hline ENS & $\operatorname{Cr}\left\{f_{5}<58\right\}=1$ & $\operatorname{Cr}\left\{f_{5}<54.293\right\}=0.9684$ \\
\hline AENS & $\operatorname{Cr}\left\{f_{6}<12.15\right\}=1$ & $\operatorname{Cr}\left\{f_{6}<11.36\right\}=0.9684$ \\
\hline
\end{tabular}

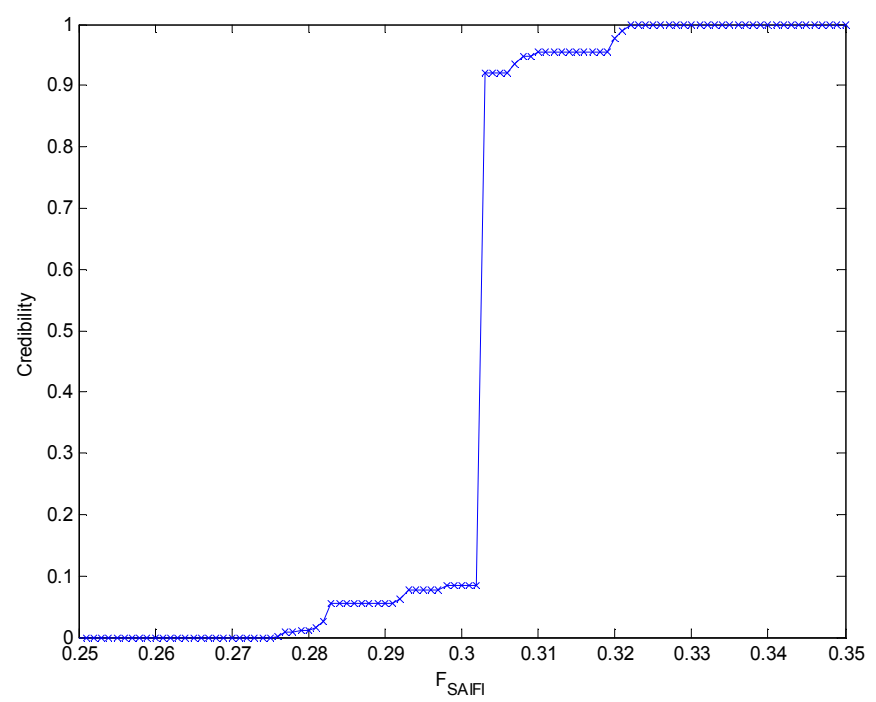

Figure 7. The relationship between $\operatorname{Cr}\left\{f_{\mathrm{SAIFI}}<F_{\mathrm{SAIFI}}\right\}$ and $F_{\text {SAIFI }}$

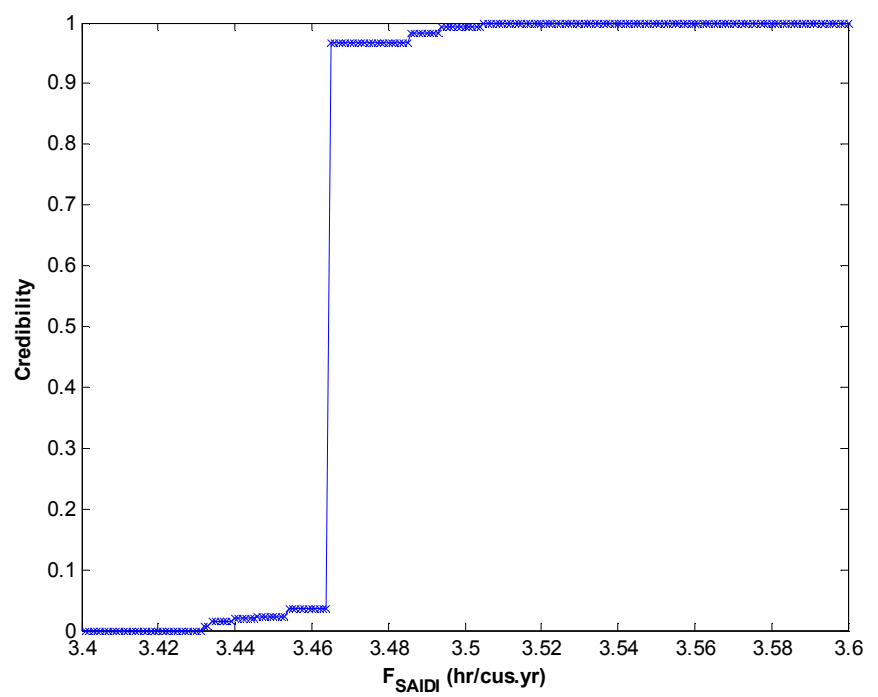

Figure 8. The relationship between $\operatorname{Cr}\left\{f_{\text {SAIDI }}<F_{\text {SAIDI }}\right\}$ and $F_{\text {SAIDI }}$ 


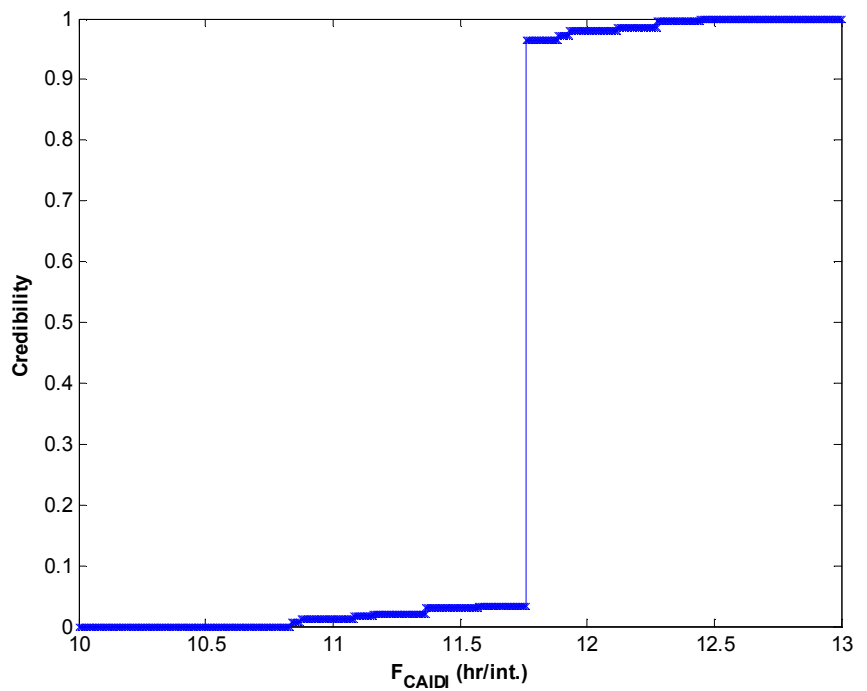

Figure 9. The relationship between $\operatorname{Cr}\left\{f_{\text {CAIDI }}<F_{\text {CAIDI }}\right\}$ and $F_{\text {CAIDI }}$

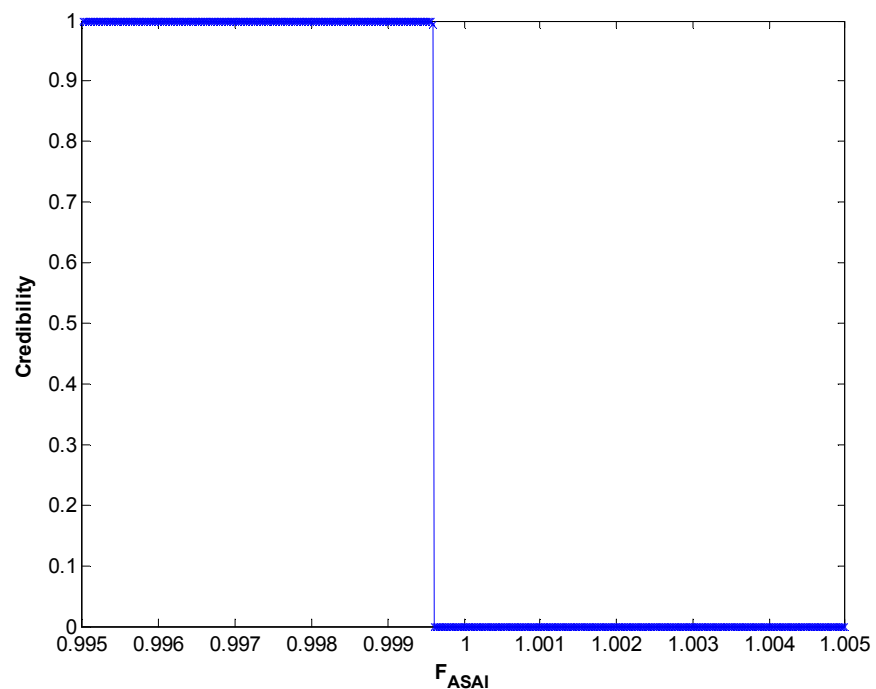

Figure 10. The relationship between $\operatorname{Cr}\left\{f_{\mathrm{ASAI}}>F_{\mathrm{ASAl}}\right\}$ and $F_{\mathrm{ASAI}}$

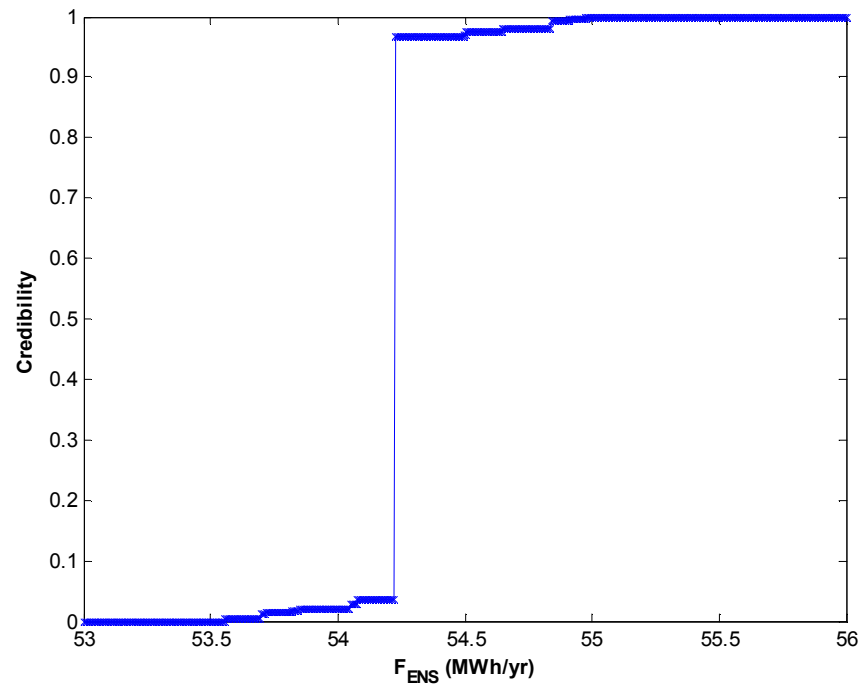

Figure 11. The relationship between $\operatorname{Cr}\left\{f_{\mathrm{ENS}}<F_{\mathrm{ENS}}\right\}$ and $F_{\mathrm{ENS}}$ 


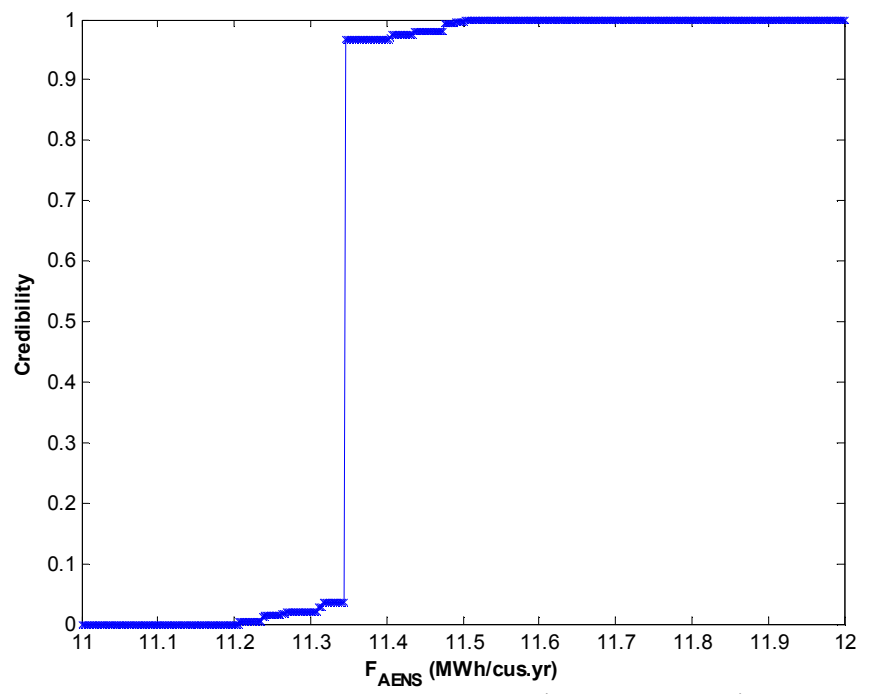

Figure 12. The relationship between $\operatorname{Cr}\left\{f_{\mathrm{AENS}}<F_{\mathrm{AENS}}\right\}$ and $F_{\mathrm{AENS}}$

\section{Conclusion}

This paper employed credibility theory to evaluate the reliability of a distribution network. This method is able to model both objective and subjective uncertainties. Compared with traditional methods, the proposed method is provides more information about the indices by showing how the indices are dispersed, rather than merely providing their expected values. This affords a better sense of realizable reliability targets, during both planning and operation. The illustrative cases have also demonstrated the feasibility and value of the approach. It is surmised that this approach based on credibility theory will afford broad application in the area of distribution system reliability evaluation.

\section{Acknowledgment}

This work was supported in part by the U.S. National Science Foundation under Grant ECCS 0702208.

\section{References}

Allan R. N., Billinton R., Sjarief I., Goel L. and So K. S., 1991. A reliability test system for education purposes - basic distribution system data and results. IEEE Transaction on Power Systems, Vol. 6, No. 2, pp. 813-820.

Atwa Y. M., Saadany E. F. E., 2009. Reliability evaluation for distribution system with renewable distributed generation during islanded mode of operation. IEEE Transactions on Power Systems, Vol. 24, No. 4, pp. 572-581.

Bae I. S., Kim J. O., 2007. Reliability evaluation of distributed generation based on operation mode. IEEE Transactions on Power Systems, Vol. 22, No. 2, pp. 785-790.

Bae I. S., Kim J. O., 2008. Reliability evaluation of customers in a microgrid. IEEE Transactions on Power Systems, Vol. 23, No. 3, pp. 1416-1422.

Billinton R., Acharya J. R., 2005. Consideration of multi-state weather models in reliability evaluation of transmission and distribution systems. Electrical and Computer Engineering, pp. 916-922.

Billinton R., Acharya J. R., 2006. Weather-based distribution system reliability evaluation. Generation, Transmission and Distribution, IEE Proceedings, Vol. 153, No. 5, pp. 499-506.

Billinton R., Allan R. N., 1996. Reliability evaluation of power systems (Second edition). Plenum press, New York, NY.

Dong L., Li J., and Bao H., 2009. Reliability evaluation of distribution system considering composite uncertainty factors. Power and Energy Engineering Conference, Asia-Pacific (APPEEC), pp. 1-5.

Feng Y. Q., Wu W. C., Zhang B. M. and Li W. Y.,2008. Power system operation risk assessment using credibility theory. IEEE Transaction on Power Systems. Vol. 23, No. 3, pp. 1309-1318.

Kennedy S., 2009. Reliability evaluation of islanded microgrids with stochastic distributed generation. Power \& Energy Society General Meeting, pp. 1-8.

Lei X. R., Ren Z., Huang W. Y. and Chen B. Y., 2005. Fuzzy reliability analysis of distribution systems accounting for parameters uncertainty. The Fourth International Conference on Machine Learning and Cybernetics, Guangzhou, China, pp. 4017-4022.

Liu B. D., 2004. Uncertainty theory: an introduction to its axiomatic foundations. Springer-Verlag, Berlin. 
Liu B. D., Liu Y. K., 2002. Expected value of fuzzy variable and fuzzy expected value models. IEEE Trans on Fuzzy Systems, Vol.10, No.4, pp.445-450.

Liu B. D., Peng J., 2005. A Course in Uncertainty Theory. Tsinghua University Press, Beijing.

Moon J. F., Kim J. C., Lee H. T., Park C. H., Yun S. Y. and Lee S. S., 2004. Reliability evaluation of distribution system through the analysis of time-varying failure rate. Power Engineering Society General Meeting, pp. 668-673.

Retterath B., Venkata S. S. and Chowdhury A. A., 2004. Impact of time-varying failure rates on distribution reliability. 8th International Conference on Probabilistic Methods Applied to Power Systems, Iowa State University, Ames, IA, pp. 953-958.

Wang H., Wu W. C., Zhang B. M., and Pan J. Y., 2007. Fuzzy power flow based on credibility theory. Automation of Electric Power Systems, Vol. 31, No. 17, pp. 21-25.

Wang P., Billinton R., 2002. Reliability cost/worth assessment of distribution systems incorporating time-varying weather conditions and restoration resources. IEEE Transactions on Power Delivery, Vol. 17, No. 1, pp. 260-265.

Yuan Y., Qian K., Zhou C., 2007. The effect of distributed generation on distribution system reliability. Universities Power Engineering Conference (UPEC), 42nd International, pp. 911-916.

\section{Biographical Notes}

Xufeng Xu was born in 1981 in Zhejiang, China. He received the B.E. degree and the Ph.D. degree in electrical engineering from Zhejiang University, Hangzhou, China. He is currently a Post-doctoral research associate in electrical engineering at Michigan State University, East Lansing, USA. His research interests include power system reliability, power system planning and microgrid architecture. E-mail: xufeng@egr.msu.edu.

Joydeep Mitra received the B.Tech. (Hons.) degree in electrical engineering from Indian Institute of Technology, Kharagpur, India, and the Ph.D. degree in electrical engineering from Texas A\&M University, College Station. He is Associate Professor of Electrical Engineering and Senior Faculty Associate of the Institute of Public Utilities at Michigan State University, East Lansing. Prior to this, he was Associate Professor at New Mexico State University, Las Cruces, Assistant Professor at North Dakota State University, Fargo, and Senior Consulting Engineer at LCG Consulting, Los Altos, CA. His research interests include power system reliability, distributed energy resources, and power system planning. E-mail: mitra@ieee.org.

Received December, 2009

Accepted March, 2010

Final acceptance in revised form March, 2010 